\title{
ZINCO E CROMO NA CICATRIZAÇÃO DE FERIDAS EM RATOS NORMAIS E DIABÉTICOS
}

\section{THE EFFECT OF ZINC AND CHROMIUM ON WOUND HEALING IN NORMAL AND DIABETIC RATS}

\author{
Sandra Pedroso de Moraes, TCBC-SP ${ }^{1}$ \\ Fátima Regina Chaves ${ }^{2}$ \\ Sérgio Banci ${ }^{3}$ \\ Patrícia A. Rover ${ }^{4}$ \\ Flávia Georgetti ${ }^{4}$ \\ José Alfredo dos Reis Neto, TCBC-SP ${ }^{5}$
}

\begin{abstract}
RESUMO: O retardo da cicatrização de feridas em pacientes diabéticos eleva os custos hospitalares e a morbidade dos procedimentos operatórios. O objetivo deste trabalho é avaliar a influência da suplementação dietética de óxido de zinco e cromo (ZC) na reparação tecidual de feridas cutâneas. Foram estudados 69 ratos Wistar, pesando entre 200-270 gramas, separados em cinco grupos (G). Na primeira semana do experimento induziu-se diabetes com streptozotocin em 53 animais (GIII, GIV, GV). A partir do $8^{\circ}$ dia os ratos normais (GI, GII) e os diabéticos receberam, além de ração e água: GI, nenhum tratamento (NT); GII, ZC adicionados à água; GIII, NT; GIV, insulina; $\mathrm{GV}, \mathrm{ZC}$ e insulina. No $15^{\circ}$ dia foi retirado fragmento elíptico de pele e subcutâneo do dorso dos animais. Nos $15^{\circ}, 22^{\circ}, 29^{\circ}$ e $36^{\circ}$ dias, as feridas operatórias foram fotografadas. Essas fotografias foram transferidas para computador, onde foram calculadas as suas áreas. Os valores obtidos foram submetidos à análise estatística. No $22^{\circ}$ dia da avaliação, não houve diferença significante (NS) na redução das áreas das feridas operatórias entre os grupos. Nas datas seguintes, os ratos diabéticos não tratados (GIII) também não apresentaram diferença significante na redução das áreas das feridas operatórias (RA) em relação aos normais (GI). Mas os tratados com zinco e cromo e com insulina (GII, GIV e GV) apresentaram RA significativamente maior do que os do GIII. O GV apresentou maior RA do que o GIV, porém NS. Conclui-se que o tratamento de ratos diabéticos com insulina e a suplementação dietética de ZC favorecem a cicatrização de feridas cutâneas.
\end{abstract}

Descritores: Zinco; Cromo; Cicatrização; Diabetes.

\section{INTRODUÇÃO}

As freqüentes complicações pós-operatórias $(\mathrm{PO})$ de pacientes diabéticos causam grande preocupação. Especialmente o metabolismo corporal e o processo de reparação tecidual encontram-se seriamente comprometidos.

O zinco, ortomolécula constituinte de diversas enzimas e parte inerente da insulina, atua no metabolismo e na cicatrização ${ }^{1,2,3,4}$. Os níveis séricos desse oligoelemento decrescem no PO e a sua suplementação torna-se necessária ${ }^{5}$. Na sua deficiência a reparação tecidual encontra-se prejudicada, com diminuição da formação de tecido conectivo ${ }^{6,7,8}$.

O cromo está intimamente ligado ao metabolismo da glicose ${ }^{9,10,11}$. Desempenha importante papel na liberação de insulina ${ }^{12} \mathrm{e}$ sua deficiência provoca alterações no metabolismo de proteínas e lipídios ${ }^{10,12}$, portanto, é fator preponderante para normalizar a utilização de glicose em pacientes diabéticos. Acredita-se que esse elemento melhore a ação e o aproveitamento do zinco no processo cicatricial ${ }^{12}$.

Não encontramos na literatura investigação sobre a ação sinérgica desses dois oligoelementos na cicatrização.

1. Doutora e Professora Titular do Departamento de Cirurgia da PUC-Campinas.

2. Doutora e Professora Titular do Departamento de Clínica Médica da PUC-Campinas.

3. Residente do Departamento de Cirurgia.

4. Alunos do Curso de Graduação em Medicina.

5. Livre Docente e Professor Titular do Departamento de Cirurgia da PUC-Campinas.

Recebido em 20/12/1999

Aceito para publicação em 2/5/2000

Trabalho realizado no Laboratório de Técnica Operatória e Cirurgia Experimental da Pontifícia Universidade Católica de Campinas - SP. 
O objetivo deste trabalho é avaliar a influência da suplementação dietética de zinco e cromo e/ou insulina no processo de reparação tecidual de feridas cutâneas em ratos normais e diabéticos.

\section{MÉTODOS}

A fase experimental foi realizada em duas etapas utilizando-se 40 ratos (etapa 1) e 29 ratos (etapa 2), Wistar, machos, pesando entre 200-270 gramas, com idade aproximada de três meses.

Quinze dias antes do início do experimento, os ratos foram levados para o Laboratório de Técnica Operatória e Cirurgia Experimental da PUC-Campinas, em gaiolas com cinco animais cada, recebendo água e ração comercial.

\section{Etapa 1}

Os animais foram separados em cinco grupos iguais (GI a GV).

$\mathrm{Na}$ primeira semana do experimento, denominada diabetogênica, 24 ratos receberam streptozotocin (STZ), para indução de diabetes, e 16 receberam soro fisiológico $0,9 \%$ (SF), na dose de 40 miligramas por quilo de peso corporal $(\mathrm{mg} / \mathrm{kg})$ e 0,15 mililitros $(\mathrm{ml})$, respectivamente, por via subcutânea (sc). Diariamente, às 7 horas, a glicemia de todos os animais era medida com glucômetro comercial. A inoculação da droga foi interrompida quando a glicemia atingiu valor igual a ou maior do que 250 miligramas/decilitro (mg/dl), por dois dias consecutivos, e o $\mathrm{SF}$, no $2^{\circ}$ dia de inoculação.

A partir do $8^{\circ}$ dia os cinco grupos, de ratos normais (GI, GII) e ratos diabéticos (GIII, GIV, GV), foram tratados diariamente, até a morte no $36^{\circ}$ dia do experimento. Receberam, além de ração e água: GI, nenhum tratamento; GII, óxido de zinco e cromo, adicionados à água, em doses aproximadas de 1,5 e $0,02 \mathrm{mg} / \mathrm{kg}$ de peso, respectivamente; GIII, nenhum tratamento; GIV, insulina NPH injetada por via sc, na dose de 2,5 unidades $/ \mathrm{kg}$, quando a glicemia variava entre $200-250 \mathrm{mg} / \mathrm{dl}$ e 3 unidades $/ \mathrm{kg}$, quando a glicemia era maior do que $250 \mathrm{mg} / \mathrm{dl}$; GV, zinco e cromo e insulina nas doses referidas para os GII e GIV, respectivamente.

No $15^{\circ}$ dia do experimento todos os ratos foram submetidos à operação asséptica. Após anestesia com éter etílico por inalação, os animais foram colocados em decúbito ventral, sobre uma prancheta com cartolina, onde tiveram suas patas fixadas e a silhueta corporal marcada. Em seguida à tricotomia dorsal, foi ressecado fragmento elíptico de pele e tecido celular subcutâneo sob molde plástico de 6 x 3,5 centímetros $(\mathrm{cm}), 2 \mathrm{~cm}$ abaixo das omoplatas e fotografada a ferida, sobre papel com escala auxiliar (papel quadriculado).

Os animais foram examinados, pesados e tratados diariamente. Nos $22^{\circ}, 29^{\circ}$ e $36^{\circ}$ dias, após colocá-los sobre as mesmas cartolinas e fixá-los na prancheta, as feridas operatórias foram também fotografadas. Nesta última data os ratos foram mortos.

\section{Etapa 2}

Repetiu-se o mesmo experimento para os grupos III (dez ratos), IV (12 ratos) e V (sete ratos). Somente modificaram-se as doses de insulina, para os GIV e GV, sendo de 1 unidade/kg, quando a glicemia variava entre $200-250 \mathrm{mg} /$ dl, 2 unidades $/ \mathrm{kg}$, quando a glicemia variava entre 250 $350 \mathrm{mg} / \mathrm{dl}$ e 3 unidades $/ \mathrm{kg}$, quando a glicemia era maior do que $350 \mathrm{mg} / \mathrm{dl}$.

Todas as fotografias das feridas operatórias foram digitalizadas com scanner colorido, 2.400 pontos por polegada de resolução máxima. Utilizou-se software destinado à obtenção de imagem digitalizada, bem como à correção de croma, contraste que evidencia os limites da cicatriz cirúrgica e o ajuste de foco. $\mathrm{O}$ arquivo foi, então, salvo em formato apropriado para compressão de dados, visando alta qualidade, e importado, para a recuperação dos limites da cicatriz por traçado manual, incluindo a captura da escala auxiliar (papel quadriculado) para a manutenção da dimensão real das feridas. $\mathrm{O}$ traçado resultante foi transferido para outro programa, onde foi feita a correção de escala e o cálculo da área e do perímetro das lesões.

A partir das medidas obtidas, calculou-se as diferenças percentuais entre os dias das operações e os dias das avaliações seguintes (D\%). Aplicou-se, então, o teste de Mann-Whitney, para comparação entre amostras independentes, duas a duas, em cada data. Fixou-se em a £ 0,05 o nível para rejeição da hipótese de nulidade.

\section{RESULTADOS}

Durante a etapa 1 do experimento morreram dois ratos do GI (25\%), dois do GII (25\%), quatro do GIII (50\%), seis do GIV (75\%) e quatro do GV (50\%). Na etapa 2 morreram seis ratos do GIII $(60 \%)$, cinco do GIV $(41,6 \%)$ e um do GV $(14,2 \%)$. Todos os óbitos ocorreram até a $4^{\circ}$ semana do estudo, sendo que não houve óbito nos do GI e do GII antes da anestesia inalatória.

À avaliação clínica, não foram encontradas alterações nos animais dos grupos I e II. Os ratos diabéticos (GIII, GIV e GV), apresentaram, progressivamente, piora do estado geral com caquexia, pêlos eriçados, distensão abdominal com silhueta de alças intestinais visíveis à inspeção, mais evidentes nos animais do GIII.

Após a primeira semana a glicemia dos ratos do GI e do GII manteve oscilação entre 80 a $110 \mathrm{mg} / \mathrm{dl}$ e a do GIII entre 200 e $500 \mathrm{mg} / \mathrm{dl}$. No GIV e no GV houve variação da glicemia, na etapa 1 , entre 25 a $500 \mathrm{mg} / \mathrm{dl}$ e 40 a $450 \mathrm{mg} / \mathrm{dl}$ e na etapa 2 entre 40 a $500 \mathrm{mg} / \mathrm{dl}$ e 45 a $276 \mathrm{mg} / \mathrm{dl}$, respectivamente.

A evolução de peso dos animais é mostrada nos Gráficos 1 e 2 .

Na etapa 1 a ingesta diária de ração e de água variou, respectivamente, entre 12 a 18 gramas $(\mathrm{g})$ e 30 a $50 \mathrm{ml}$ no GI e no GII, 10 a $40 \mathrm{~g}$ e 100 a $150 \mathrm{ml}$ no GIII, 18 a $40 \mathrm{~g}$ e 90 a $100 \mathrm{ml}$ no GIV e 16 a $33 \mathrm{~g}$ e 40 a $100 \mathrm{ml}$ no GV. 


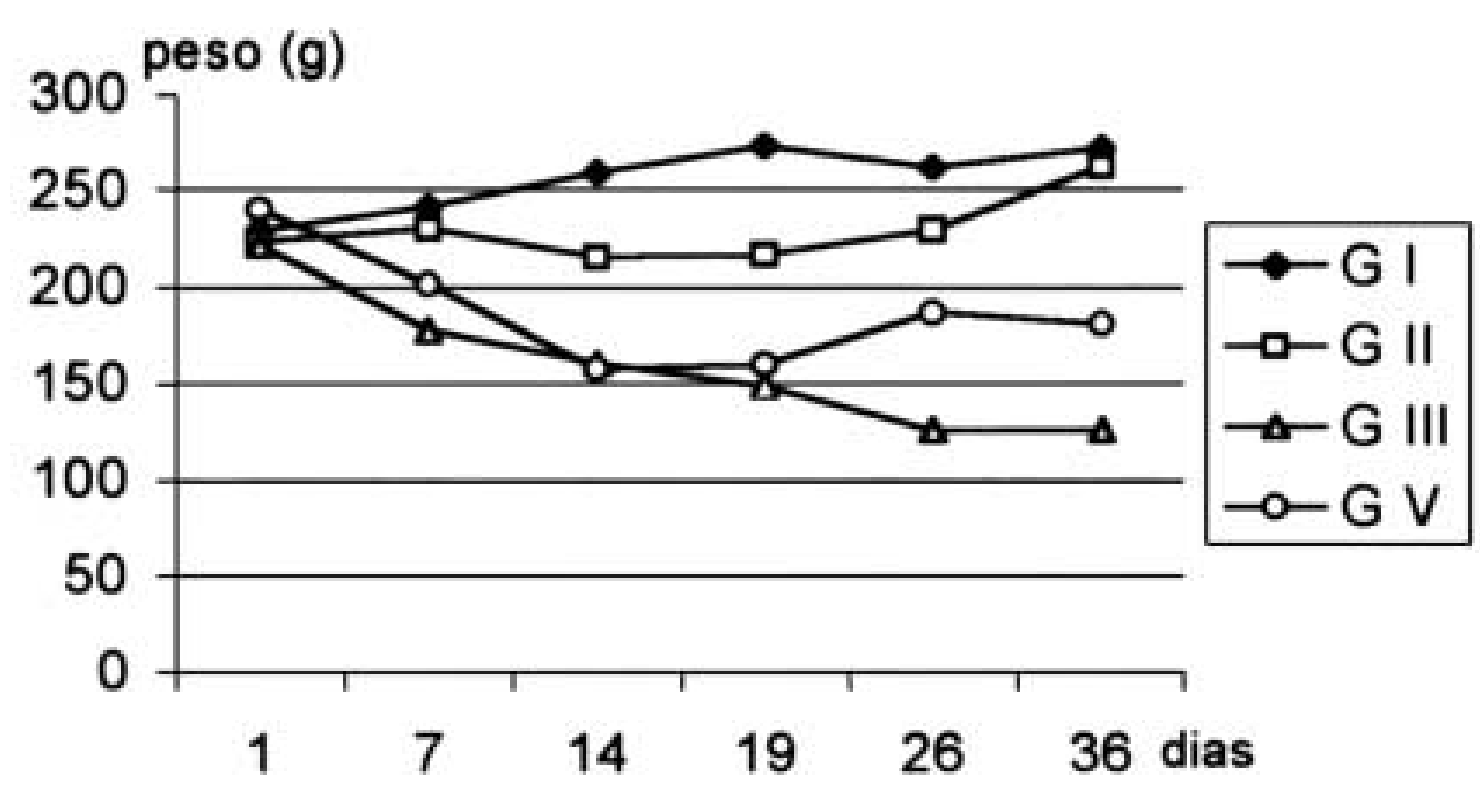

Gráfico 1 - Peso médio dos ratos dos quatro grupos estudados na etapa 1, nas datas acima especificadas.

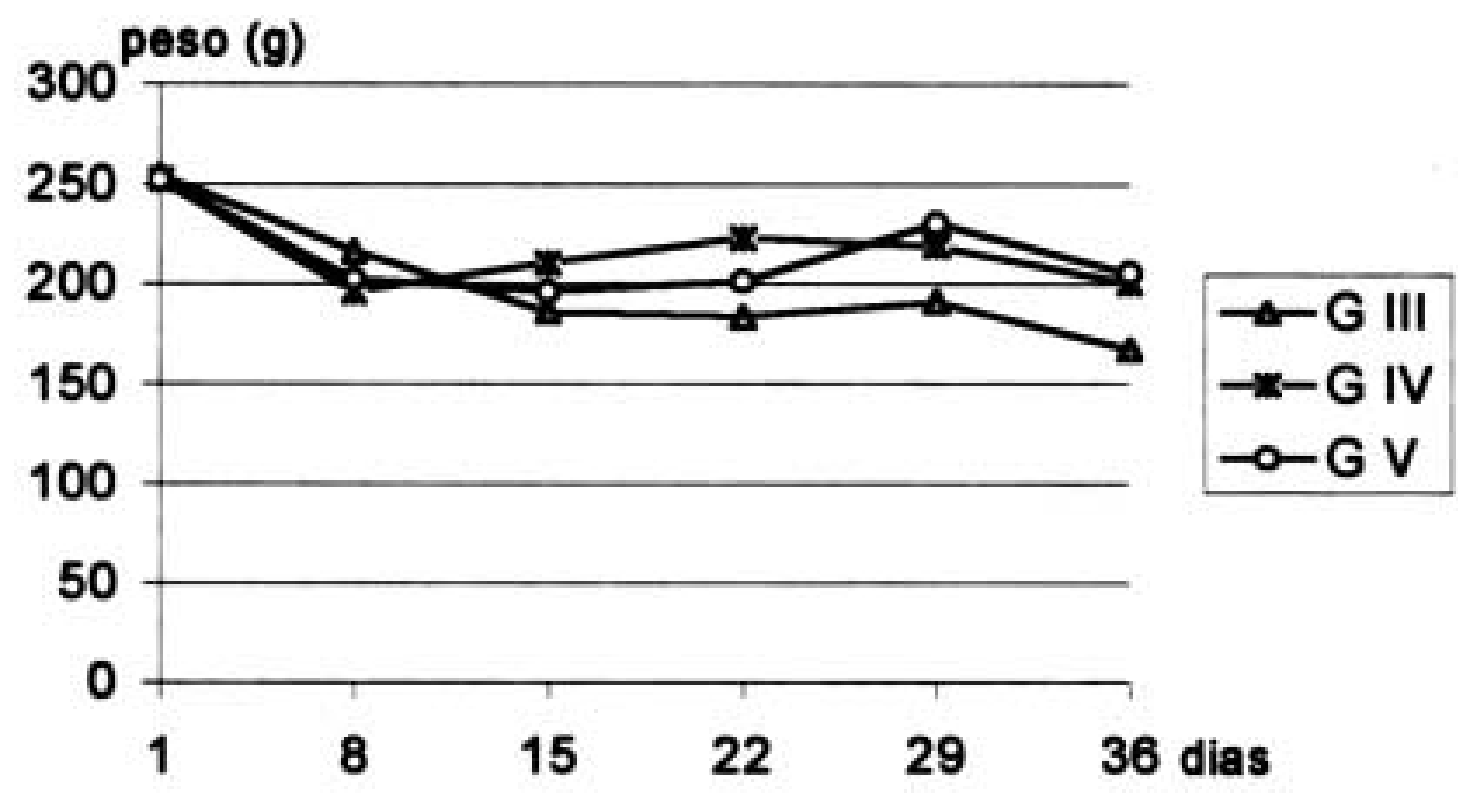

Gráfico 2 - Peso médio dos ratos dos três grupos estudados na etapa 2, nas datas acima especificadas.

A avaliação das feridas operatórias mostrou:

No $22^{\circ}$ dia do experimento, não havia diferença na percentagem de regressão de área das feridas operatórias. No $29^{\circ}$ dia, o GII apresentou percentagem de regressão significativamente maior do que o GIII $(p=0,03)$. No $36^{\circ}$ dia, o GII e o GV apresentaram percentagem de regressão significativamente maior do que o GIII ( $\mathrm{p}=0,03$ e $\mathrm{p}=0,02$; respectivamente). $\mathrm{O}$ reduzido número de ratos do GIV, nas datas de avaliação das feridas, não permitiu estudo estatístico. Os resultados dessa etapa encontram-se no Gráfico 3. 
No $22^{\circ}$ dia do experimento, não havia diferença na percentagem de regressão de área das feridas operatórias. No $29^{\circ}$ dia os GIV e GV apresentaram percentagem de regressão de área das feridas significativamente maior do que o GIII ( $\mathrm{p}=0,03$ e $\mathrm{p}=0,01$; respectivamente). O mesmo ocorreu no $36^{\circ}$ dia $(\mathrm{p}=0,006$ e $\mathrm{p}=0,008$; respectivamente). A média dos valores está representada no Gráfico 4.

\section{DISCUSSÃO}

Grande parte dos recursos da saúde é gasta no tratamento de complicações metabólicas de pacientes diabéticos ${ }^{13}$. Muitas pesquisas estão sendo desenvolvidas para melhorar a qualidade de vida desses pacientes mas não encontramos estudos sobre o efeito da suplementação dietética do zinco associado ao cromo no controle

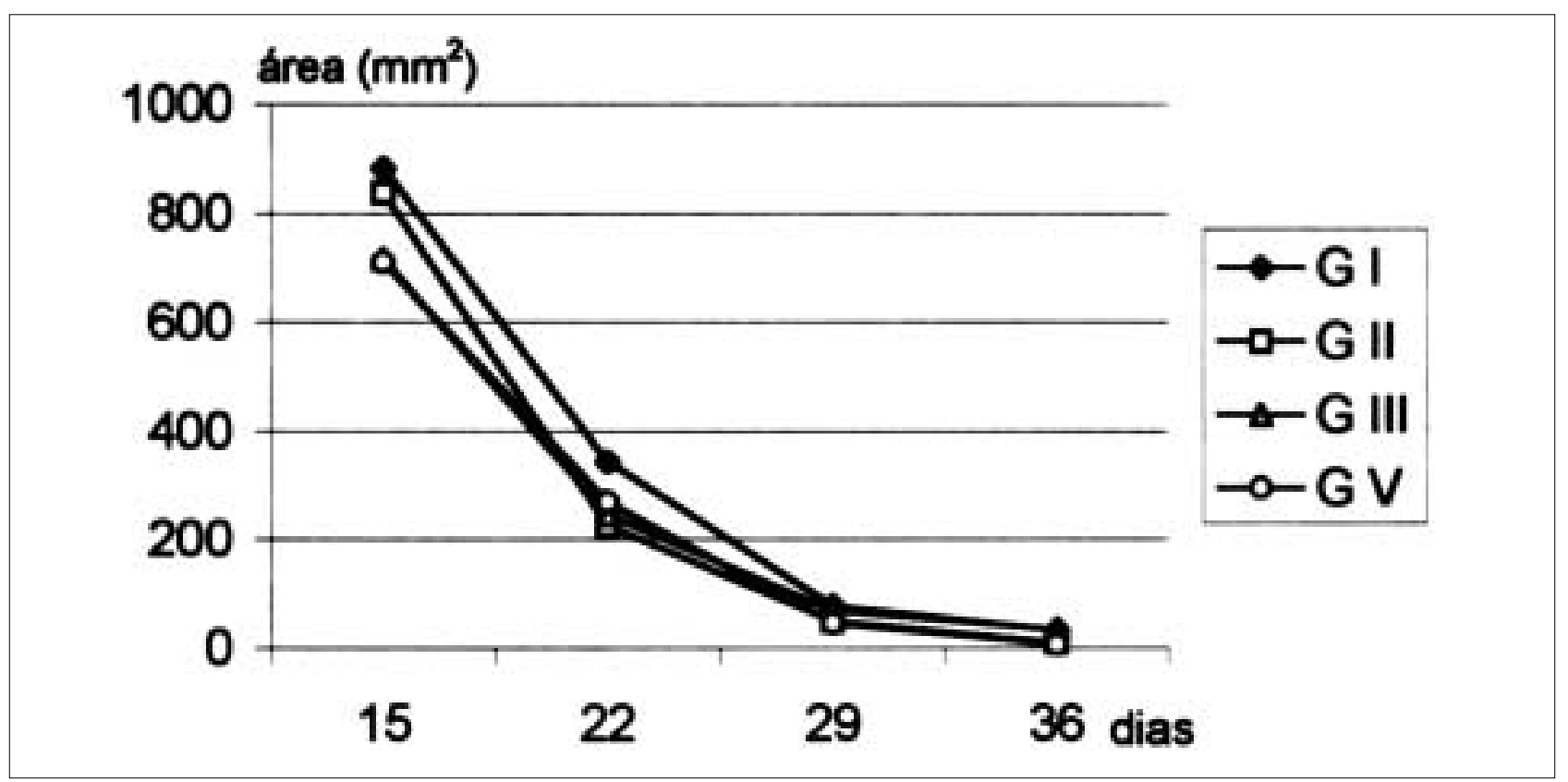

Gráfico 3 - Área média das feridas operatórias dos quatro grupos de ratos estudados na etapa 1, nas datas acima especificadas.

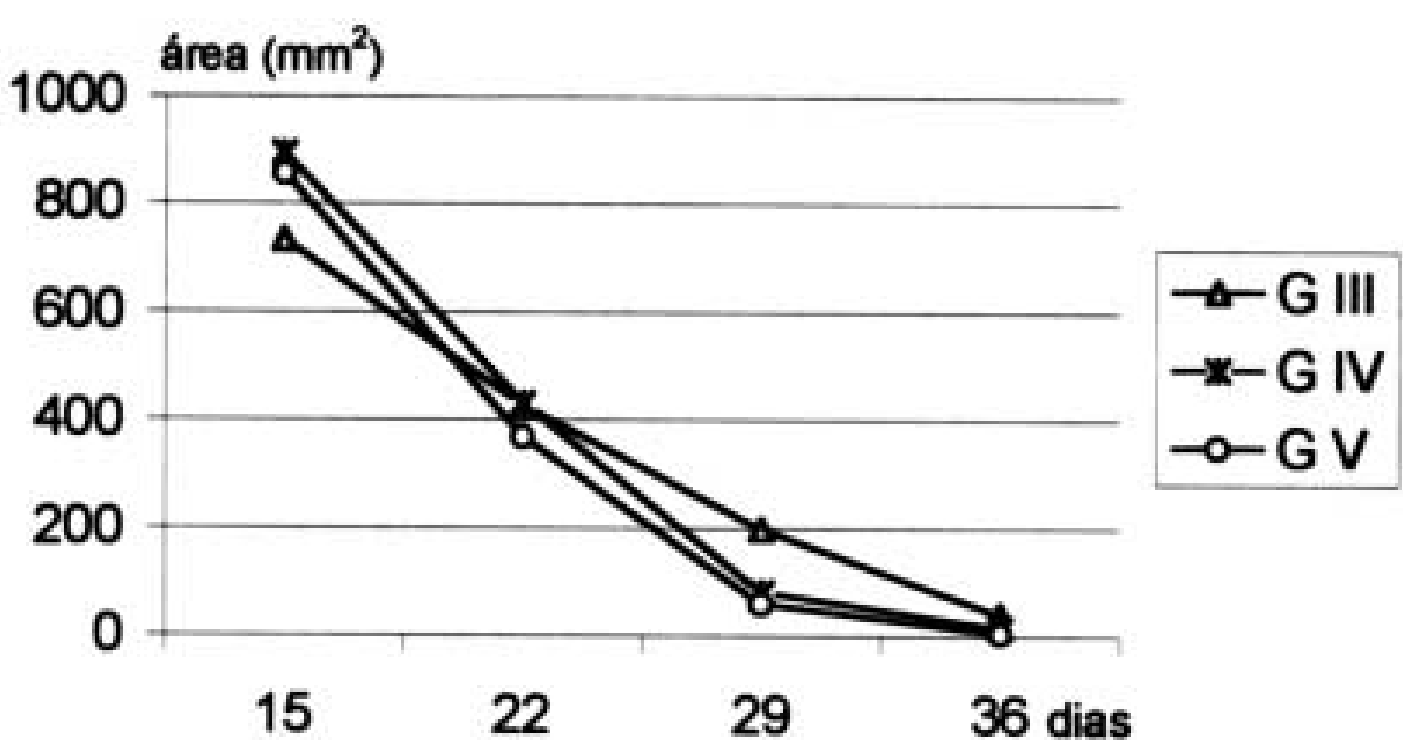

Gráfico 4 - Área média das feridas operatórias dos três grupos de ratos estudados na etapa 2, nas datas acima especificadas. 
dessas complicações metabólicas e na cicatrização de feridas.

Para avaliar a reparação tecidual cutânea induziu-se diabetes com streptozotocin em ratos Wistar, modelo bem estudado ${ }^{14-26}$.

Decidiu-se realizar outra etapa do trabalho em razão da grande mortalidade, durante a indução do diabetes e pelo tratamento com insulina na etapa 1 . As doses preconizadas dessa droga, freqüentemente provocaram hipoglicemia e morte dos ratos, o que tornou necessária sua modificação na etapa 2. Nessa, houve predominância de hiperglicemia, mesmo tomando-se o cuidado de infundir a insulina sempre às 18 horas, ou seja, no período pré-prandial.

Nas duas etapas os ratos com suplementação dietética com ZC apresentaram menor índice de mortalidade, provavelmente em razão do menor desequilíbrio metabólico.

Os óbitos verificados nos ratos do GI e do GII ocorreram por depressão respiratória, durante anestesia inalatória com éter, nos dias de avaliação das feridas operatórias. Assim como em alguns animais dos outros grupos.

As alterações clínicas encontradas nos ratos diabéticos, inclusive a dilatação de alças intestinais, têm sido relatada por outros autores ${ }^{26}$.

Somente os animais do GI e do GII ganharam peso durante a evolução do trabalho. Nos ratos diabéticos, a perda de peso foi menor nos grupos tratados com insulina e, principalmente, quando houve a adição de zinco e cromo à dieta.

Os animais diabéticos geralmente ingeriram maior quantidade de alimento e água. Mas o consumo dos ratos do GIII oscilou entre ingestão abaixo da normalidade até aumento de três vezes, em relação ao rato normal.

$\mathrm{O}$ tratamento com insulina e, principalmente, a inclusão do zinco e do cromo, mantiveram estável o volume da ingesta e favoreceram o equilíbrio do metabolismo com melhora do estado nutricional, portanto esses micronutrientes atuam no equilíbrio metabólico dos animais.

A literatura tem dado grande ênfase para o zinco como elemento importante na reconstrução de tecido cicatricial ${ }^{4}$. O uso tópico deste, em feridas cutâneas apresenta resultados controversos ${ }^{2,3,27}$. A deficiência de zinco na dieta de ratos provocou diminuição da síntese protéica e da formação de tecido cicatricial em lesões de mesentério de intestino delgado ${ }^{7}$.

Quanto ao cromo, sua ingesta excessiva, provocou efeitos tóxicos em ratos $^{28}$. Não se conhece as necessidades orgânicas desse micronutriente, mas sabe-se que sua deficiência em humanos e em animais superiores, ocasiona transtornos no metabolismo da glicose e sintomas similares aos do diabetes ${ }^{12}$.

O cromo também atua no metabolismo de lipídios, na síntese de proteínas e provavelmente no metabolismo dos ácidos nucléicos em humanos ${ }^{8,9,10}$. Em ratas, um dos estudos constatou diminuição da tolerância à glicose endoveno$\mathrm{sa}$, deficiência de crescimento, aumento do colesterol sérico que reverteu com a suplementação dietética de cromo $^{29}$.

No presente trabalho, quando foram comparados os grupos de ratos não diabéticos, em todas as datas estudadas, houve maior retração cicatricial nas feridas dos animais que receberam os micronutrientes adicionados à dieta, mas sem significância estatística.

No $22^{\circ}$ dia da avaliação não houve diferença significante entre os grupos, na retração cicatricial das feridas. Até essa data provavelmente predominaram os fenômenos inflamatórios da primeira fase da cicatrização.

A partir das datas das avaliações seguintes, os ratos diabéticos não tratados (GIII) apresentaram retração cicatricial menor em relação aos outros grupos. Quando se comparou com os ratos normais (GI), não apresentou significância estatística, provavelmente pelo número reduzido de animais estudados. Mas quando se comparou com os GII, GIV e, principalmente com o GV, havia grande diferença, com significância estatística. Esse último grupo apresentou maior retração cicatricial do que o GIV, porém sem significância estatística no número de ratos avaliados.

Pelos resultados encontrados, a associação de zinco e cromo na dieta parece ter importante papel na cicatrização, entretanto outros estudos deverão ser realizados, incluindo-se outros métodos de avaliação.

Conclui-se que a suplementação dietética de zinco e cromo e o tratamento de ratos diabéticos com insulina, favorecem a cicatrização de feridas cutâneas.

\begin{abstract}
The delay in tissue repair of cutaneous wounds in diabetic patients increase hospital costs and morbidity in surgical procedures. The purpose of this study is to assess the influence of zinc oxide and chromium as diet supplements (ZC) in tissue repair of cutaneous wounds. This study was carried out in 69 Wistar rats with weight ranging from 200 to 270 grams divided in 5 (five) groups $(G)$. Diabetes was induced in 53 animals with streptozotocin in the first week of the study (GIII, GIV, and GV). After the 8th day, normal (GI, GII) and diabetic rats (GIII, GIV, GV) received diet supplements together with food and water: GI, no treatment (NT); GII, ZC added to the water; GIII, NT; GIV, insulin; GV, ZC and insulin. On the 15th day an elliptical fragment was taken from the skin and from the dorsum of the animals. The operative wounds were then photographed on days 15, 22, 29 and 36. These pictures were sent to a computer and wound areas were calculated. The scores obtained were then subjected to statistical analysis. On the 22nd day of assessment there was no significant difference (NS) in the reduction of the operative wound site among the groups (RA). In the following dates the diabetic rats that did not receive the treatment (GII) also did not have any significant difference in RA in comparison to normal rats (GI). On the other hand, those rats treated with zinc, chromium and insulin (GII, GIV. and GV) had a higher RA level than those of the GIII did. The GV had a higher level of RA than GIV, but it was not significant. (NS). The conclusion was that the treatment of diabetic rats with insulin and diet supplement with ZC improved the healing of cutaneous wounds.
\end{abstract}

Key Words: Zinc, Chromium, Wound healing, Diabetes. 


\section{REFERÊNCIAS}

1. Agren MS - Studies on zinc in wound healing. Acta Derm. Venereol. Suppl. Stockh 1990; 154: 1-36.

2. Agren MS, Chvapil M, Franzén L - Enhancement of re-epithelialization with topical zinc oxide in porcine partial-thickness wounds. J. Surg. Res. 1991; 50: 101-5.

3. Agren MS, Franzén L, Chvapil M - Effects on wound healing of zinc oxide in a hydrocolloid dressing. J. Am. Acad. Dermatol. 1993; 29: 221-7.

4. Lansdown ABG - Zinc in the healing wound. Lancet 1996; 347: 706-7.

5. Faure H, Peyrin JC, Richard MJ et al - Parenteral supplementation on with zinc in surgical patients corrects postoperative serum-zinc drop. Biol. Trace Elem. Res. 1991; 30: 35-7.

6. Agren MS \& Franzen L - Influence of zinc deficiency on breaking strenght of 3-week-old skin incisions in the rat. Acta Chir. Scand. 1990; 156: 667-70.

7. Franzén LE \& Ghassemifar MR - Connective tissue repair in zinc deficiency. Eur. J. Surg. 1992; 158: 333-7.

8. Pino CG, Treche MH, Ferrer RS et al - Niveles sericos de cromo, cobre y cinc en un grupo de niños obesos. Rev. Cubana Aliment. Nutr. 1992; 6: 94-8.

9. Mahdi GS - Chromium deficiency might contribute to insulin resistance, type 2 diabetes mellitus, dyslipidaemia, and atherosclerosis. Diabetic Medicine 1996; 13: 38990.

10. Anderson RA, Cheng N, Bryden NA et al - Elevated intakes of supplemental chromium improve glucose and insulin variables in individuals with type 2 diabetes. Diabetes 1997; 46: 1786-91.

11. Kimura K - Role of essential trace elements in the disturbance of carbohydrate metabolism. Japanese J. Clin. Med. 1996; 54: 79.

12. Baran EJ - Algunos comentarios sobre la bioquímica del cromo y el factor de tolerancia a la glucosa. Acta Bioq. Clin. Latinoamericana 1986; 20: 191-4.

13. Herman WH, Dasbach EJ, Songer TJ et al - The cost - effetiveness of intensive therapy for diabetes mellitus. Endocrinol. Metab. Clin. North Am. 1997; 26:679-95.

14. Rerup CC - Drugs producing diabetes through damage of the insulin secreting cells. Pharmacol. Rev. 1970; 22:485-518.

15. Greene DA, De Jesus Jr PV, Winegrad AI - Effects of insulin and dietary myoinositol on impaired peripheral motor nerve conduction velocity in acute streptozotocin diabetes. J. Clin. Invest. 1975; 55:1326-36.

16. Penpargkul S, Schaible T, Yipintsoi $\mathrm{T}$ et al - The effect of diabetes on performance and metabolism of rat hearts. Circ. Res. 1980; 47:901-21.

17. Fein FS, Kornstein LB, Strobeck JE et al - Altered myocardial mechanics in diabetic rats. Circ. Res.1980; 47:922-33.

18. Raw I, Juliani MH, Rocha MC et al - Effect of insulin on the synthesis of rat liver ribosomal and endoplasmic reticulum proteins. Brasilian J. Med. Biol. Res. 1985; 18:421-6.

19. Kusaka M, Kishi K, Sokabe H - Does so-called streptozotocin hypertencion exists in rats? Hypertension 1987; 10:517-21.

20. Edelstein D \& Brownlee M - Aminoguanidine ameliorates albuminuria in diabetic hypertensive rats. Diabetologia $1992 ; 35: 96-7$.
21. Catanzaro OL, Marina-Prendes MG, Hope SI et al - Streptozotocin-induced hyperglycemia is decreased by nitric oxide inhibition. Brazilian J. Med. Biol. Res. 1994; 27:2043-7.

22. Hammes HP, Brownlee M, Edelstein D et al - Aminoguanidine inhibits the development of accelerated diabetic retinopathy in the spontaneous hypertensive rat. Diabetologia 1994; 37:32-5.

23. Ralevic V, Belai A, Burnstock G - Effects of streptozotocindiabetes on sympathetic nerv, endothelial and smooth muscle function in the rat mesenteric arterial bed. Eur. J. Pharmacol. 1995; 286:193-9.

24. Martinez BB, Marumo CK, Leão CS et al - Renal effect of gentamicin in diabetic rats. Brazilian J. Med. Biol. Res. 1995; 28:801-4.

25. Pepato MT, Migliorini RH, Goldberg AL et al - Role of different proteolytic pathways in degradation of muscle protein from streptozotocin-diabetic rats. Am. J. Physiol. 1996; 271:E340-7.

26. Kam KL, Hendriks MGC, Pijl AJ et al - Contractile responses to various stimuli in isolated resistance vessels from simultaneously hypertensive and streptozotocin-diabetic rats. J. Cardiovasc. Pharmacol. 1996; 27:167-75.

27. Agren MS, Söderberg TA, Reuterving C et al - Effect of topical zinc oxide on bacterial growth and inflammation in full-thickness skin wounds in normal and diabetics rats. Eur. J. Surg. 1991; 157:97-101.

28. O'Flaherty EJ - A physiologically based model of chromium kinetics in the rat. Toxicol. Appl. Pharmacol. 1996; 138:54-64.

29. Mertz W, Toepfer EW, Roginski EE et al - Present knowledge of the role of chromium. Federat. Proc. 1974; $33: 2275-2280$.

Endereço para correspondência:

Dra. Sandra Pedroso de Moraes

Rua Boaventura do Amaral, 684/131

13015-191 Campinas - São Paulo

Fones: (19) 3236-0436, 3232-2335

E-mail: grapas@ nutecnet.com.br 